\title{
The Hyers-Ulam-Rassias stability of the quartic functional equation in fuzzy $\beta$-normed spaces
}

\author{
Xiuzhong Yang ${ }^{1,2}$, Guannan Shen ${ }^{1,2}$, Guofen Liu ${ }^{1,2^{*}}$ and Lidan Chang ${ }^{1,2}$
}

\section{"Correspondence:}

liugf2003@163.com

${ }^{1}$ College of Mathematics and

Information Science, Hebei Normal

University, Shijiazhuang, 050024,

People's Republic of China

${ }^{2}$ Hebei Key Laboratory of

Computational Mathematics and

Applications, Shijiazhuang, 050024,

People's Republic of China

\begin{abstract}
In this article, we extend general fuzzy normed spaces to fuzzy $\beta$-normed spaces and adopt the fixed point and direct methods to prove the Hyers-Ulam-Rassias stability of the quartic functional equation $f(2 x+y+z)+f(2 x+y-z)+f(2 x-y+z)+f(-2 x+y+$ $z)+f(2 y)+f(2 z)=8[f(x+y)+f(x-y)+f(x+z)+f(x-z)]+2[f(y+z)+f(y-z)]+32 f(x)$ in fuzzy $\beta$-normed spaces.
\end{abstract}

MSC: 39B82; 47H10; 47S40

Keywords: Hyers-Ulam-Rassias stability; fuzzy $\beta$-normed space; quartic functional equation; fixed point

\section{Introduction}

In 1940, Ulam [1] asked the following question concerning the stability of group homomorphisms: Under what condition is there an additive mapping near an approximately additive mapping between a group and a metric group?

In the next year, Hyers [2] gave a positive answer to the above question for additive groups under the assumption that the groups are Banach spaces. In 1978, Rassias [3] extended the theorem of Hyers by considering the unbounded Cauchy difference. Găvruta [4] proved the Hyers-Ulam-Rassias stability with the generalized control function. This stability result is called the Hyers-Ulam-Rassias stability of functional equations. Rassias [5] first introduced the quartic functional equation

$$
f(x+2 y)+f(x-2 y)+6 f(x)=4[f(x+y)+f(x-y)+6 f(y)]
$$

and solved the Ulam stability problem of the function. Later Sahoo and Chung [6], and Lee et al. [7] remodified Rassias' equation and obtained its general solution.

Pinsker [8] characterized orthogonally additive functionals on an inner product space. The orthogonal Cauchy functional equation

$$
f(x+y)=f(x)+f(y), \quad x \perp y
$$

(c) 2015 Yang et al. This article is distributed under the terms of the Creative Commons Attribution 4.0 International License (http://creativecommons.org/licenses/by/4.0/), which permits unrestricted use, distribution, and reproduction in any medium, provided you give appropriate credit to the original author(s) and the source, provide a link to the Creative Commons license, and indicate if changes were made. 
in which $\perp$ is an abstract orthogonality relation, was first investigated by Gudder and Strawther [9]. Ger and Sikorska discussed the orthogonal stability of the orthogonal Cauchy functional equation in [10].

Park [11] proved the Hyers-Ulam-Rassias stability of the orthogonality quartic functional equation

$$
f(x+2 y)+f(x-2 y)+6 f(x)=4[f(x+y)+f(x-y)+6 f(y)], \quad x \perp y
$$

where $\perp$ is the orthogonality in the sense of Rätz.

Let us recall the orthogonality in the sense of Rätz [12].

Definition 1.1 ([9]) Suppose $X$ is a real vector space with $\operatorname{dim} X \geq 2$ and $\perp$ is a binary relation on $X$ with the following properties:

$\left(\mathrm{O}_{1}\right)$ totality of $\perp$ for zero: $x \perp 0,0 \perp x$ for all $x \in X$;

$\left(\mathrm{O}_{2}\right)$ independence: if $x, y \in X-\{0\}$, then $x, y$ are linearly independent;

$\left(\mathrm{O}_{3}\right)$ homogeneity: if $x, y \in X, x \perp y$, then $\alpha x \perp \beta y$ for all $\alpha, \beta \in \mathbb{R}$;

$\left(\mathrm{O}_{4}\right)$ the Thalesian property: if $P$ is a 2 -dimensional subspace of $X, x \in P$ and $\lambda \in \mathbb{R}_{+}$, which is the set of nonnegative real numbers, then there exists $y_{0} \in P$ such that $x \perp y_{0}$ and $x+y_{0} \perp \lambda x-y_{0}$.

The pair $(X, \perp)$ is called an orthogonality space. By an orthogonality normed space we mean an orthogonality space having a normed structure.

Arunkumar et al. [13] proved that a mapping $f: X \rightarrow Y$ satisfies the functional equation

$$
\begin{aligned}
& f(2 x+y+z)+f(2 x+y-z)+f(2 x-y+z)+f(-2 x+y+z)+f(2 y)+f(2 z) \\
& \quad=8[f(x+y)+f(x-y)+f(x+z)+f(x-z)]+2[f(y+z)+f(y-z)]+32 f(x)
\end{aligned}
$$

if and only if the mapping $f: X \rightarrow Y$ is quartic. Moreover, they proved the Hyers-UlamRassias stability of the quartic functional equation (1.1) in orthogonality normed spaces.

Katsaras [14] defined a fuzzy norm on a vector space to construct a fuzzy vector topological structure on the space. Some mathematicians have defined fuzzy norms of a vector space from various points of view (see [15-20]).

Bag and Samanta [21], following Cheng and Mordeson [22], gave an idea of fuzzy norm in such a manner that the corresponding fuzzy metric is of Karmosil and Michalek [23].

A number of mathematicians have extensively investigated the stability problems of several functional equations, and they obtained many interesting results concerning the problem (for instance [24-38]).

In this article, we extend general fuzzy normed spaces to fuzzy $\beta$-normed spaces and prove the Hyers-Ulam-Rassias stability of the orthogonally quartic functional equation (1.1) in this kind of spaces by two different methods: the fixed point and direct methods.

Now we introduce the concept of a fuzzy $\beta$-normed vector space and associated concept of a fuzzy $\beta$-norm, followed by some examples to show the validity of the notion.

Definition 1.2 Let $\mathrm{X}$ be a real vector space. A function $N_{\beta}: X \times \mathbb{R} \rightarrow[0,1]$ is called a fuzzy $\beta$-norm on $X$ with $0<\beta \leq 1$ if for all $x, y \in X$ and $s, t \in \mathbb{R}$, 
(N1) $N_{\beta}(x, t)=0$ for $t \leq 0$;

(N2) $x=0$ if and only if $N_{\beta}(x, t)=1$ for all $t>0$;

(N3) $N_{\beta}(c x, t)=N_{\beta}\left(x, \frac{t}{|c|^{\beta}}\right)$ if $c \neq 0$;

(N4) $N_{\beta}(x+y, s+t) \geq \min \left\{N_{\beta}(x, s), N_{\beta}(y, t)\right\}$;

(N5) $N_{\beta}(x, \cdot)$ is a non-decreasing function of $\mathbb{R}$ and $\lim _{t \rightarrow \infty} N_{\beta}(x, t)=1$;

(N6) for $x \neq 0, N_{\beta}(x, \cdot)$ is continuous on $\mathbb{R}$.

The pair $\left(X, N_{\beta}\right)$ is called a fuzzy $\beta$-normed vector space.

We remark that when $\beta=1,\left(X, N_{\beta}\right)$ is fuzzy normed space $(X, N)$.

Example 1.3 Let $\left(X,\|\cdot\|_{\beta}\right)(0<\beta \leq 1)$ be a $\beta$-normed linear space and $\alpha, \gamma>0$. Then

$$
N_{\beta}(x, t)= \begin{cases}\frac{\alpha t}{\alpha t+\gamma\|x\|_{\beta}}, & t>0, x \in X \\ 0, & t \leq 0, x \in X\end{cases}
$$

is a fuzzy $\beta$-norm on $X$.

Proof (N1), (N2), and (N6) are obviously true.

Notice that for any $t \in \mathbb{R}, t>0$, and $c \neq 0$

$$
N_{\beta}(c x, t)=\frac{\alpha t}{\alpha t+\gamma\|c x\|_{\beta}}=\frac{\alpha t}{\alpha t+\gamma|c|^{\beta}\|x\|_{\beta}}=\frac{\alpha \cdot \frac{t}{|c|^{\beta}}}{\alpha \cdot \frac{t}{|c|^{\beta}}+\gamma\|x\|_{\beta}}=N_{\beta}\left(x, \frac{t}{|c|^{\beta}}\right),
$$

which implies that (N3) holds.

To prove (N4), let $s>0, t>0$, we assume that $N_{\beta}(x, s) \geq N_{\beta}(y, t)$, thus

$$
\begin{aligned}
N_{\beta}(x, s)-N_{\beta}(y, t) & =\frac{\alpha s}{\alpha s+\gamma\|x\|_{\beta}}-\frac{\alpha t}{\alpha t+\gamma\|y\|_{\beta}} \\
& =\frac{\alpha s\left(\alpha t+\gamma\|y\|_{\beta}\right)-\alpha t\left(\alpha s+\gamma\|x\|_{\beta}\right)}{\left(\alpha s+\gamma\|x\|_{\beta}\right)\left(\alpha t+\gamma\|y\|_{\beta}\right)} \\
& =\frac{\alpha \gamma s\|y\|_{\beta}-\alpha \gamma t\|x\|_{\beta}}{\left(\alpha s+\gamma\|x\|_{\beta}\right)\left(\alpha t+\gamma\|y\|_{\beta}\right)} \\
& \geq 0,
\end{aligned}
$$

we can get

$$
\alpha \gamma\left(s\|y\|_{\beta}-t\|x\|_{\beta}\right) \geq 0 .
$$

We have by the above inequality

$$
\begin{aligned}
N_{\beta}(x+y, s+t)-N_{\beta}(y, t) & =\frac{\alpha(s+t)}{\alpha(s+t)+\gamma\|x+y\|_{\beta}}-\frac{\alpha t}{\alpha t+\gamma\|y\|_{\beta}} \\
& \geq \frac{\alpha(s+t)}{\alpha(s+t)+\gamma\|x\|_{\beta}+\gamma\|y\|_{\beta}}-\frac{\alpha t}{\alpha t+\gamma\|y\|_{\beta}} \\
& =\frac{\alpha(s+t)\left(\alpha t+\gamma\|y\|_{\beta}\right)}{\left[\alpha(s+t)+\gamma\|x\|_{\beta}+\gamma\|y\|_{\beta}\right]\left(\alpha t+\gamma\|y\|_{\beta}\right)}
\end{aligned}
$$




$$
\begin{aligned}
& -\frac{\alpha t\left[\alpha(s+t)+\gamma\|x\|_{\beta}+\gamma\|y\|_{\beta}\right]}{\left[\alpha(s+t)+\gamma\|x\|_{\beta}+\gamma\|y\|_{\beta}\right]\left(\alpha t+\gamma\|y\|_{\beta}\right)} \\
= & \frac{\alpha \gamma\left(s\|y\|_{\beta}-t\|x\|_{\beta}\right)}{\left[\alpha(s+t)+\gamma\|x\|_{\beta}+\gamma\|y\|_{\beta}\right]\left(\alpha t+\gamma\|y\|_{\beta}\right)} \\
\geq & 0 .
\end{aligned}
$$

So,

$$
N_{\beta}(x+y, s+t) \geq N_{\beta}(y, t) .
$$

Similarly, from $N_{\beta}(y, t) \geq N_{\beta}(x, s)$, we can obtain

$$
N_{\beta}(x+y, s+t) \geq N_{\beta}(x, s) .
$$

Therefore, $N_{\beta}(x+y, s+t) \geq \min \left\{N_{\beta}(x, s), N_{\beta}(y, t)\right\}$ and (N4) hold.

For any $t_{1}, t_{2} \in \mathbb{R}$, and $t_{1} \geq t_{2}$,

$$
\begin{aligned}
N_{\beta}\left(x, t_{1}\right)-N_{\beta}\left(x, t_{2}\right) & =\frac{\alpha t_{1}}{\alpha t_{1}+\gamma\|x\|_{\beta}}-\frac{\alpha t_{2}}{\alpha t_{2}+\gamma\|x\|_{\beta}} \\
& =\frac{\alpha t_{1}\left(\alpha t_{2}+\gamma\|x\|_{\beta}\right)-\alpha t_{2}\left(\alpha t_{1}+\gamma\|x\|_{\beta}\right)}{\left(\alpha t_{1}+\gamma\|x\|_{\beta}\right)\left(\alpha t_{2}+\gamma\|x\|_{\beta}\right)} \\
& =\frac{\left(t_{1}-t_{2}\right) \alpha \gamma\|x\|_{\beta}}{\left(\alpha t_{1}+\gamma\|x\|_{\beta}\right)\left(\alpha t_{2}+\gamma\|x\|_{\beta}\right)} \geq 0 .
\end{aligned}
$$

So $N_{\beta}(x, \cdot)$ is a non-decreasing function of $\mathbb{R}$, which proves (N5).

Definition 1.4 Let $\left(X, N_{\beta}\right)$ be a fuzzy $\beta$-normed vector space. A sequence $\left\{x_{n}\right\}$ in $X$ is said to be convergent or converge if there exists an $x \in X$ such that $\lim _{n \rightarrow \infty} N_{\beta}\left(x_{n}-x, t\right)=1$ for all $t>0$. In this case, $x$ is called the limit of the sequence $\left\{x_{n}\right\}$ in $X$ and we denote $N_{\beta}$ $\lim _{n \rightarrow \infty} x_{n}=x$.

Definition 1.5 Let $\left(X, N_{\beta}\right)$ be a fuzzy $\beta$-normed vector space. A sequence $\left\{x_{n}\right\}$ in $X$ is called Cauchy if for each $\varepsilon>0$ and each $t>0$ there exists an $n_{0} \in \mathbb{N}$ such that for all $n \geq n_{0}$ and all $p>0$, we have $N_{\beta}\left(x_{n+p}-x_{n}, t\right)>1-\varepsilon$.

It is well known that every convergent sequence in a fuzzy $\beta$-normed vector space is Cauchy. If each Cauchy sequence is convergent, then the fuzzy $\beta$-norm is said to be complete and the fuzzy $\beta$-normed vector space is called a complete fuzzy $\beta$-normed space.

Example 1.6 Let $N: X \times \mathbb{R} \rightarrow[0,1]$ be a fuzzy $\beta$-norm on $\mathbb{R}$ defined by

$$
N_{\beta}(x, t)= \begin{cases}\frac{t}{t+|x|^{\beta}}, & t>0 \\ 0, & t \leq 0\end{cases}
$$

Then $\left(\mathbb{R}, N_{\beta}\right)$ is a complete fuzzy $\beta$-normed space. 
Proof Let $\left\{x_{n}\right\}$ be a Cauchy sequence in $\mathbb{R}, \delta>0$, and $\varepsilon=\frac{\delta^{\beta}}{1+\delta^{\beta}}$. Then there exist some $m \in \mathbb{N}$ such that for all $n \geq m$ and all $p>0$,

$$
\frac{1}{1+\left|x_{n+p}-x_{n}\right|^{\beta}} \geq 1-\varepsilon
$$

So $\left|x_{n+p}-x_{n}\right|^{\beta}<\delta$ for all $n \geq m$ and all $p>0$. Therefore $\left\{x_{n}\right\}$ is a Cauchy sequence in $\left(\mathbb{R},|\cdot|^{\beta}\right)$. Let $x_{n} \rightarrow x_{0} \in \mathbb{R}$ as $n \rightarrow \infty$. Then $\lim _{n \rightarrow \infty} N_{\beta}\left(x_{n}-x_{0}, t\right)=1$ for all $t>0$. The rest of the proof is similar to the proof of Example 1.3.

Definition 1.7 Let $X$ be a set. A function $d: X \times X \rightarrow[0, \infty]$ is called a generalized metric on $X$ if and only if $d$ satisfies

(M1) $d(x, y)=0$ if and only if $x=y$;

(M2) $d(x, y)=d(y, x)$ for all $x, y \in X$;

(M3) $d(x, z) \leq d(x, y)+d(y, z)$ for all $x, y, z \in X$.

Theorem 1.8 ([7]) Let $(X, d)$ be a generalized complete metric space and $J: X \rightarrow X$ be a strictly contractive mapping with Lipschitz constant $L<1$. Then, for all $x \in X$, either

$$
d\left(J^{n} x, J^{n+1} x\right)=\infty
$$

for all nonnegative integers $n$ or there exists a positive integer $n_{0}$ such that

(i) $d\left(J^{n} x, J^{n+1} x\right)<\infty$ for all $n \geq n_{0}$;

(ii) the sequence $\left\{J^{n} x\right\}$ converges to a fixed point $x^{*}$ of $J$;

(iii) $x^{*}$ is the unique fixed point of $J$ in the set $X^{*}=\left\{y \in X \mid d\left(J^{n_{0}} x, y\right)<\infty\right\}$;

(iv) $d\left(y, x^{*}\right) \leq \frac{1}{1-L} d(J y, y)$ for all $y \in X^{*}$.

Definition 1.9 An even mapping $f: X \rightarrow Y$ is called an orthogonally quartic mapping if

$$
\begin{aligned}
& f(2 x+y+z)+f(2 x+y-z)+f(2 x-y+z)+f(-2 x+y+z)+f(2 y)+f(2 z) \\
& \quad=8[f(x+y)+f(x-y)+f(x+z)+f(x-z)]+2[f(y+z)+f(y-z)]+32 f(x)
\end{aligned}
$$

for all $x, y, z \in X$ with $x \perp y, y \perp z$ and $z \perp x$ in the sense of Rätz.

\section{Fuzzy stability of the orthogonally quartic functional equation: fixed point method}

In this section, using the fixed point method, we prove the Hyers-Ulam-Rassias stability of the quartic functional equation in complete fuzzy $\beta$-normed spaces.

Definition 2.1 Let $(X, \perp)$ be a real orthogonality vector space and $\left(Y, N_{\beta}\right)$ be a complete fuzzy $\beta$-normed space, where $0<\beta \leq 1$. Define a difference operator $D f: X^{3} \rightarrow Y$ by

$$
\begin{aligned}
D f(x, y, z)= & f(2 x+y+z)+f(2 x+y-z)+f(2 x-y+z)+f(-2 x+y+z)+f(2 y) \\
& +f(2 z)-8[f(x+y)+f(x-y)+f(x+z)+f(x-z)] \\
& -2[f(y+z)+f(y-z)]-32 f(x)
\end{aligned}
$$

for all $x, y, z \in X$ with $x \perp y, y \perp z$, and $z \perp x$ in the sense of Rätz. 
Theorem 2.2 Let $(X, \perp)$ be a real orthogonality vector space, $\left(Y, N_{\beta}\right)$ be a complete fuzzy $\beta$ normed space with $0<\beta \leq 1$, and $\varphi: X^{3} \rightarrow[0, \infty)$ be a function and there exists a constant $L, 0<L<1$, such that

$$
\varphi(2 x, 2 y, 2 z) \leq 16^{\beta} L \varphi(x, y, z)
$$

for all $x, y, z \in X$, with $x \perp y, y \perp z$, and $z \perp x$. Assume that a mapping $f: X \rightarrow Y$ is an even mapping satisfying $f(0)=0$ and the inequality

$$
N_{\beta}(D f(x, y, z), t) \geq \frac{t}{t+\varphi(x, y, z)}
$$

for all $x, y, z \in X, t>0$, with $x \perp y, y \perp z$, and $z \perp x$. Then there is a unique orthogonally quartic mapping $Q: X \rightarrow Y$ such that

$$
N_{\beta}(f(x)-Q(x), t) \geq \frac{16^{\beta}(1-L) t}{16^{\beta}(1-L) t+\varphi(0, x, 0)}
$$

for all $x \in X, t>0$.

Proof From (2.1), we get

$$
\varphi\left(2^{n} x, 2^{n} y, 2^{n} z\right) \leq 16^{n \beta} L^{n} \varphi(x, y, z)
$$

for all $x, y, z \in X$, with $x \perp y, y \perp z$, and $z \perp x$. Replacing $(x, y, z)$ by $(0, x, 0)$ in $(2.2)$, we get

$$
N_{\beta}(f(2 x)-16 f(x), t) \geq \frac{t}{t+\varphi(0, x, 0)}
$$

for all $x \in X$ and all $t>0$. From (N3), we get

$$
N_{\beta}\left(\frac{1}{16} f(2 x)-f(x), \frac{1}{16^{\beta}} t\right) \geq \frac{t}{t+\varphi(0, x, 0)}
$$

for all $x \in X$ and all $t>0$.

If we define

$$
E=\{h: X \rightarrow Y \mid h(0)=0\}
$$

and introduce a generalized metric on $E$ as follows:

$$
d(g, h)=\inf \left\{\mu \in[0, \infty] \mid N_{\beta}(g(x)-h(x), \mu t) \geq \frac{t}{t+\varphi(0, x, 0)}, x \in X, t>0\right\},
$$

then $(E, d)$ is complete (see[18]).

We define an operator $J: E \rightarrow E$ by

$$
(J h)(x)=\frac{1}{16} h(2 x)
$$

for all $x \in X$. 
First, we assert that $J$ is strictly contractive on $E$. Given $g, h \in E$, let $d(g, h)=\alpha$. Then

$$
N_{\beta}(g(x)-h(x), \alpha t) \geq \frac{t}{t+\varphi(0, x, 0)}
$$

for all $x \in X$ and all $t>0$. Hence

$$
\begin{aligned}
N_{\beta}((g g)(x)-(J h)(x), \alpha L t) & =N_{\beta}\left(\frac{1}{16} g(2 x)-\frac{1}{16} h(2 x), \alpha L t\right) \\
& =N_{\beta}\left(g(2 x)-h(2 x), 16^{\beta} \alpha L t\right) \\
& \geq \frac{16^{\beta} L t}{16^{\beta} L t+\varphi(0,2 x, 0)} \\
& \geq \frac{t}{t+\varphi(0, x, 0)}
\end{aligned}
$$

for all $x \in X$ and all $t>0$, so, $d(J g, J h) \leq \alpha L$. Then we conclude that $d(J g, J h) \leq L d(g, h)$ for all $g, h \in E$.

Next, we assert that $d(J f, f)<\infty$. From (2.6), we get

$$
N_{\beta}\left((J f)(x)-f(x), \frac{1}{16^{\beta}} t\right) \geq \frac{t}{t+\varphi(0, x, 0)}
$$

for all $x \in X$ and all $t>0$, i.e.,

$$
d(J f, f) \leq \frac{1}{16^{\beta}}<\infty
$$

Now, it follows from Theorem 1.9(ii) that there exists a function $Q: X \rightarrow Y$ with $Q(0)=$ 0 , which is a fixed point of $J($ i.e., $J(2 x)=16 J(x))$, such that $J^{n} f \rightarrow Q$, namely,

$$
Q(x)=\lim _{n \rightarrow \infty} \frac{f\left(2^{n} x\right)}{16^{n}}
$$

for all $x \in X$.

Since the integer $n_{0}$ of Theorem 1.8(i) is 0 and $f \in E^{*}$ (see Theorem 1.8(iii) for the definition of $X^{*}$ ), by (iv) of Theorem 1.8 and (a), we get

$$
d(f, Q) \leq \frac{1}{1-L} d(J f, f) \leq \frac{1}{16^{\beta}} \frac{1}{1-L}
$$

for all $x \in X$. So,

$$
N_{\beta}\left(f(x)-Q(x), \frac{1}{16^{\beta}} \frac{1}{1-L} t\right) \geq \frac{t}{t+\varphi(0, x, 0)},
$$

then the inequality (2.3) is true for all $x \in X, t>0$.

From (2.1) and (2.2) we have

$$
N_{\beta}\left(\frac{1}{16^{n}} D f\left(2^{n} x, 2^{n} y, 2^{n} z\right), \frac{t}{16^{n \beta}}\right) \geq \frac{t}{t+\varphi\left(2^{n} x, 2^{n} y, 2^{n} z\right)}
$$


for all $x, y, z \in X, t>0$ with $x \perp y, y \perp z$, and $z \perp x$ and $n \in \mathbb{N}$, since $2^{n} x \perp 2^{n} y, 2^{n} y \perp 2^{n} z$, and $2^{n} z \perp 2^{n} x$. So, from (2.4), we get

$$
N_{\beta}\left(\frac{1}{16^{n}} D f\left(2^{n} x, 2^{n} y, 2^{n} z\right), t\right) \geq \frac{16^{n \beta} t}{16^{n \beta} t+16^{n \beta} L^{n} \varphi(x, y, z)},
$$

then

$$
\begin{aligned}
N_{\beta}( & \frac{1}{16^{n}} f\left(2^{n}(2 x+y+z)\right)+\frac{1}{16^{n}} f\left(2^{n}(2 x+y-z)\right)+\frac{1}{16^{n}} f\left(2^{n}(2 x-y+z)\right) \\
& +\frac{1}{16^{n}} f\left(2^{n}(-2 x+y+z)\right)+\frac{1}{16^{n}} f\left(2^{n}(2 y)\right)+\frac{1}{16^{n}} f\left(2^{n}(2 z)\right) \\
& -8\left[\frac{1}{16^{n}} f\left(2^{n}(x+y)\right)+\frac{1}{16^{n}} f\left(2^{n}(x-y)\right)+\frac{1}{16^{n}} f\left(2^{n}(x+z)\right)+\frac{1}{16^{n}} f\left(2^{n}(x-z)\right)\right] \\
& \left.-2\left[\frac{1}{16^{n}} f\left(2^{n}(y+z)\right)+\frac{1}{16^{n}} f\left(2^{n}(y-z)\right)\right]-32 \times \frac{1}{16^{n}} f\left(2^{n}(x)\right), t\right) \\
\geq & \frac{t}{t+L^{n} \varphi(x, y, z)}
\end{aligned}
$$

for all $x, y, z \in X, t>0$ with $x \perp y, y \perp z$, and $z \perp x$ and $n \in \mathbb{N}$. Since

$$
\lim _{n \rightarrow \infty} \frac{t}{t+L^{n} \varphi(x, y, z)}=1
$$

for all $x, y, z \in X, t>0$ with $x \perp y, y \perp z$, and $z \perp x$, we obtain

$$
\begin{aligned}
& Q(2 x+y+z)+Q(2 x+y-z)+Q(2 x-y+z)+Q(-2 x+y+z)+Q(2 y)+Q(2 z) \\
& \quad=8[Q(x+y)+Q(x-y)+Q(x+z)+Q(x-z)]+2[Q(y+z)+Q(y-z)]+32 Q(x)
\end{aligned}
$$

for all $x, y, z \in X, t>0$ with $x \perp y, y \perp z$, and $z \perp x$.

Assume that the inequality (2.3) is also satisfied with another quartic function $Q^{\prime}: X \rightarrow$ $Y$ besides $Q$. $Q^{\prime}$ satisfies $Q^{\prime}(x)=(1 / 16) Q^{\prime}(2 x)=\left(J Q^{\prime}\right)(x)$ for all $x \in X$, and $Q^{\prime}$ is a fixed point of $J$. From (3.3) and the definition of $d$, we can get

$$
d\left(f, Q^{\prime}\right) \leq \frac{1}{16^{\beta}} \frac{1}{1-L}<\infty
$$

then

$$
d\left(J f, Q^{\prime}\right) \leq d(J f, f)+d\left(f, Q^{\prime}\right) \leq \frac{1}{16^{\beta}}+\frac{1}{16^{\beta}} \frac{1}{1-L}<\infty .
$$

So, $Q^{\prime} \in E^{*}=\{y \in E \mid d(J f, y)<\infty\}$. (In view of (a), the integer $n_{0}$ of Theorem 1.8(i) is 0 .) Thus, Theorem 1.8(ii) implies that $Q=Q^{\prime}$. This proves the uniqueness of $Q$. The proof is complete.

Corollary 2.3 Let $\theta \geq 0, p$ be a real positive number with $p<4$, and $(X, \perp)$ be a real orthogonality vector space with $\beta$-norm $\|\cdot\|_{\beta}$ with $0<\beta \leq 1$. Assume that $f: X \rightarrow Y$ is an 
even mapping satisfying $f(0)=0$ and the inequality

$$
N_{\beta}(D f(x, y, z), t) \geq \frac{t}{t+\theta\left(\|x\|_{\beta}^{p}+\|y\|_{\beta}^{p}+\|z\|_{\beta}^{p}\right)}
$$

for all $x, y, z \in X, t>0$, with $x \perp y, y \perp z$, and $z \perp x$. Then there is a unique orthogonally quartic mapping $Q: X \rightarrow Y$ such that

$$
N_{\beta}(f(x)-Q(x), t) \geq \frac{\left(16^{\beta}-2^{p \beta}\right) t}{\left(16^{\beta}-2^{p \beta}\right) t+\theta\|x\|_{\beta}^{p}}
$$

for all $x \in X, t>0$.

Proof From Theorem 2.2 by taking $\varphi(x, y, z)=\theta\left(\|x\|_{\beta}^{p}+\|y\|_{\beta}^{p}+\|z\|_{\beta}^{p}\right)$ for all $x, y, z \in X, t>0$, with $x \perp y, y \perp z$, and $z \perp x$, and choosing $L=\left(\frac{2^{p}}{16}\right)^{\beta}$ we can get the desired result.

Theorem 2.4 Let $(X, \perp)$ be a real orthogonality vector space and $\left(Y, N_{\beta}\right)$ be a complete fuzzy $\beta$-normed space with $0<\beta \leq 1$. Assume that $\varphi: X^{3} \rightarrow[0, \infty)$ is a function and there exists a constant $L, 0<L<1$, such that

$$
\varphi\left(\frac{x}{2}, \frac{y}{2}, \frac{z}{2}\right) \leq \frac{L}{16^{\beta}} \varphi(x, y, z)
$$

for all $x, y, z \in X$, with $x \perp y, y \perp z$, and $z \perp x$. If a mapping $f: X \rightarrow Y$ is an even mapping satisfying $f(0)=0$ and (2.2) for all $x, y, z \in X, t>0$, with $x \perp y, y \perp z$, and $z \perp x$, there is a unique orthogonally quartic mapping $Q: X \rightarrow Y$ such that

$$
N_{\beta}(f(x)-Q(x), t) \geq \frac{16^{\beta}\left(L^{-1}-1\right) t}{16^{\beta}\left(L^{-1}-1\right) t+\varphi(0, x, 0)}
$$

for all $x \in X, t>0$.

Corollary 2.5 Let $\theta \geq 0$ and $p$ be a real positive number with $p>4$ and $(X, \perp)$ be a real orthogonality vector space with $\beta$-norm $\|\cdot\|_{\beta}$ with $0<\beta \leq 1$. Iff $: X \rightarrow Y$ is an even mapping satisfying $f(0)=0$ and (2.7) for all $x, y, z \in X, t>0$, with $x \perp y, y \perp z$, and $z \perp x$, there is a unique orthogonally quartic mapping $Q: X \rightarrow Y$ such that

$$
N_{\beta}(f(x)-Q(x), t) \geq \frac{\left(2^{p \beta}-16^{\beta}\right) t}{\left(2^{p \beta}-16^{\beta}\right) t+\theta\|x\|_{\beta}^{p}}
$$

for all $x \in X, t>0$.

\section{Fuzzy stability of the orthogonally quartic functional equation: direct method}

In this section, we assume that $(X, \perp)$ is a real orthogonality vector space, $\left(Y, N_{\beta}\right)$ is a complete fuzzy $\beta$-normed space with $0<\beta \leq 1$ and $\left(Z, N_{\beta}^{\prime}\right)$ is a fuzzy $\beta$-normed space.

Theorem 3.1 Assume that a mapping $f: X \rightarrow Y$ is an even mapping satisfying the inequality

$$
N_{\beta}(D f(x, y, z), t) \geq N_{\beta}^{\prime}(\varphi(x, y, z), t)
$$


and $f(0)=0$ for all $x, y, z \in X, t>0$, with $x \perp y, y \perp z$, and $z \perp x$, and $\varphi: X^{3} \rightarrow Z$ is a mapping such that

$$
N_{\beta}^{\prime}\left(\varphi\left(\frac{x}{2}, \frac{y}{2}, \frac{z}{2}\right), t\right) \geq N_{\beta}^{\prime}\left(\varphi(x, y, z), \frac{t}{\alpha^{\beta}}\right)
$$

for some constant $\alpha \in \mathbb{R}$ with $0<\alpha<\frac{1}{16}$, and all $x, y, z \in X, t>0$, with $x \perp y, y \perp z$ and $z \perp x$. Then there is a unique orthogonally quartic mapping $Q: X \rightarrow Y$ such that

$$
N_{\beta}(f(x)-Q(x), t) \geq N_{\beta}^{\prime}\left(\varphi(0, x, 0),\left(\alpha^{-\beta}-16^{\beta}\right) t\right)
$$

for all $x \in X, t>0$.

Proof It follows from (3.2) that

$$
N_{\beta}^{\prime}\left(\varphi\left(\frac{x}{2^{j}}, \frac{y}{2^{j}}, \frac{z}{2^{j}}\right), t\right) \geq N_{\beta}^{\prime}\left(\varphi(x, y, z), \frac{t}{\alpha^{\beta j}}\right)
$$

for all $x, y, z \in X, t>0$, and any integers $j \geq 0$, with $x \perp y, y \perp z$, and $z \perp x$. Replacing $(x, y, z)$ by $(0, x, 0)$ in (3.1), Since $0 \perp x$, we get

$$
N_{\beta}(f(2 x)-16 f(x), t) \geq N_{\beta}^{\prime}(\varphi(0, x, 0), t)
$$

for all $x \in X$ and all $t>0$. Replacing $x$ by $\frac{x}{2}$ in (3.5)

$$
N_{\beta}\left(f(x)-16 f\left(\frac{x}{2}\right), t\right) \geq N_{\beta}^{\prime}\left(\varphi\left(0, \frac{x}{2}, 0\right), t\right)
$$

for all $x \in X$ and all $t>0$. Replacing $x$ by $\frac{x}{2 j}$ in (3.6)

$$
N_{\beta}\left(f\left(\frac{x}{2^{j}}\right)-16 f\left(\frac{x}{2^{j+1}}\right), t\right) \geq N_{\beta}^{\prime}\left(\varphi\left(0, \frac{x}{2^{j+1}}, 0\right), t\right)
$$

for all $x \in X$ and all $t>0$. Now from (N3) and (3.7), we get

$$
N_{\beta}\left(16^{j} f\left(\frac{x}{2^{j}}\right)-16^{j+1} f\left(\frac{x}{2^{j+1}}\right), 16^{\beta j} t\right) \geq N_{\beta}^{\prime}\left(\varphi\left(0, \frac{x}{2^{j+1}}, 0\right), t\right)
$$

for all $x \in X$ and all $t>0$. It follows from (3.4) that

$$
N_{\beta}\left(16^{j} f\left(\frac{x}{2^{j}}\right)-16^{j+1} f\left(\frac{x}{2^{j+1}}\right), 16^{\beta j} t\right) \geq N_{\beta}^{\prime}\left(\varphi(0, x, 0), \frac{t}{\alpha^{\beta(j+1)}}\right)
$$

for all $x \in X$ and all $t>0$, namely,

$$
N_{\beta}\left(16^{j} f\left(\frac{x}{2^{j}}\right)-16^{j+1} f\left(\frac{x}{2^{j+1}}\right), 16^{\beta j} \alpha^{\beta(j+1)} t\right) \geq N_{\beta}^{\prime}(\varphi(0, x, 0), t)
$$


for all $x \in X$ and all $t>0$. So

$$
\begin{aligned}
& N_{\beta}\left(16^{n} f\left(\frac{x}{2^{n}}\right)-f(x), \sum_{j=0}^{n-1} 16^{\beta j} \alpha^{\beta(j+1)} t\right) \\
& \quad=N_{\beta}\left(\sum_{j=0}^{n-1}\left(16^{j+1} f\left(\frac{x}{2^{j+1}}\right)-16^{j} f\left(\frac{x}{2^{j}}\right)\right), \sum_{j=0}^{n-1} 16^{\beta j} \alpha^{\beta(j+1)} t\right) \\
& \quad \geq \min _{0 \leq j \leq n-1}\left\{N_{\beta}\left(16^{j+1} f\left(\frac{x}{2^{j+1}}\right)\right)-16^{j} f\left(\frac{x}{2^{j}}\right), 16^{\beta j} \alpha^{\beta(j+1)} t\right\} \\
& \geq N_{\beta}^{\prime}(\varphi(0, x, 0), t),
\end{aligned}
$$

which yields

$$
\begin{aligned}
& N_{\beta}\left(16^{n+p} f\left(\frac{x}{2^{n+p}}\right)-16^{p} f\left(\frac{x}{2^{p}}\right), \sum_{j=0}^{n-1} 16^{\beta(p+1)} \alpha^{\beta(j+p+1)} t\right) \\
& \quad=N_{\beta}\left(\sum_{j=0}^{n-1}\left(16^{p+j} f\left(\frac{x}{2^{p+j}}\right)-16^{p+j+1} f\left(\frac{x}{2^{p+j+1}}\right)\right), \sum_{j=0}^{n-1} 16^{\beta(p+j)} \alpha^{\beta(p+j+1)} t\right) \\
& \geq \min _{0 \leq j \leq n-1}\left\{N_{\beta}\left(16^{p+j} f\left(\frac{x}{2^{p+j}}\right)\right)-16^{p+j+1} f\left(\frac{x}{2^{p+j+1}}\right), 16^{\beta(p+j)} \alpha^{\beta(p+j+1)} t\right\} \\
& \geq N_{\beta}^{\prime}(\varphi(0, x, 0), t)
\end{aligned}
$$

for all $x \in X, t>0$, and $n>0, p \geq 0$. So,

$$
N_{\beta}\left(16^{n+p} f\left(\frac{x}{2^{n+p}}\right)-16^{p} f\left(\frac{x}{2^{p}}\right), t\right) \geq N_{\beta}^{\prime}\left(\varphi(0, x, 0), \frac{t}{\sum_{j=0}^{n-1} 16^{\beta(p+j)} \alpha^{\beta(p+j+1)}}\right)
$$

for all $x \in X, t>0$, and $n>0, p \geq 0$. Since the series $\sum_{j=0}^{n-1} 16^{\beta j} \alpha^{\beta j}$ is convergent, we see by taking the limit $p \rightarrow \infty$ in the last inequality that the sequence $\left\{16^{n} f\left(\frac{x}{2^{n}}\right)\right\}$ is a Cauchy sequence in the complete fuzzy $\beta$-normed space $\left(Y, N_{\beta}\right)$, so it converges in $Y$. We define a mapping $Q: X \rightarrow Y$ by $Q(x)=N_{\beta}-\lim _{n \rightarrow \infty} 16^{n} f\left(\frac{x}{2^{n}}\right)$ for all $x \in X$. It means that

$$
\lim _{n \rightarrow \infty} N_{\beta}\left(Q(x)-16^{n} f\left(\frac{x}{2^{n}}\right), t\right)=1
$$

for all $x \in X, t>0$.

Replacing $x, y, z$ by $2^{n} x, 2^{n} y, 2^{n} z$ in (3.1), respectively, we get

$$
N_{\beta}\left(D f\left(\frac{x}{2^{n}}, \frac{y}{2^{n}}, \frac{z}{2^{n}}\right), t\right) \geq N_{\beta}^{\prime}\left(\varphi\left(\frac{x}{2^{n}}, \frac{y}{2^{n}}, \frac{z}{2^{n}}\right), t\right)
$$

for all $x, y, z \in X, t>0$, with $x \perp y, y \perp z$, and $z \perp x$ and all $n \in \mathbb{N}$. Since $\frac{x}{2^{n}} \perp \frac{y}{2^{n}}, \frac{y}{2^{n}} \perp \frac{z}{2^{n}}$, and $\frac{z}{2^{n}} \perp \frac{x}{2^{n}}$, we have

$$
N_{\beta}\left(16^{n} D f\left(\frac{x}{2^{n}}, \frac{y}{2^{n}}, \frac{z}{2^{n}}\right), 16^{n \beta} t\right) \geq N_{\beta}^{\prime}\left(\varphi\left(\frac{x}{2^{n}}, \frac{y}{2^{n}}, \frac{z}{2^{n}}\right), t\right),
$$


namely,

$$
N_{\beta}\left(16^{n} D f\left(\frac{x}{2^{n}}, \frac{y}{2^{n}}, \frac{z}{2^{n}}\right), t\right) \geq N_{\beta}^{\prime}\left(\varphi\left(\frac{x}{2^{n}}, \frac{y}{2^{n}}, \frac{z}{2^{n}}\right), \frac{t}{16^{n \beta}}\right)
$$

for all $x, y, z \in X, t>0$, with $x \perp y, y \perp z$, and $z \perp x$ and all $n \in \mathbb{N}$. From (3.4), we get

$$
N_{\beta}\left(16^{n} D f\left(\frac{x}{2^{n}}, \frac{y}{2^{n}}, \frac{z}{2^{n}}\right), t\right) \geq N_{\beta}^{\prime}\left(\varphi(x, y, z), \frac{t}{(16 \alpha)^{n \beta}}\right)
$$

for all $x, y, z \in X, t>0$, with $x \perp y, y \perp z$, and $z \perp x$ and all $n \in \mathbb{N}$. So

$$
\begin{aligned}
N_{\beta}( & 16^{n} f\left(\frac{2 x+y+z}{2^{n}}\right)+16^{n} f\left(\frac{2 x-y+z}{2^{n}}\right)+16^{n} f\left(\frac{2 x-y+z}{2^{n}}\right) \\
& +16^{n} f\left(\frac{-2 x+y+z}{2^{n}}\right)-8\left[16^{n} f\left(\frac{x+y}{2^{n}}\right)+16^{n} f\left(\frac{x-y}{2^{n}}\right)\right. \\
& \left.+16^{n} f\left(\frac{x+z}{2^{n}}\right)+16^{n} f\left(\frac{x-z}{2^{n}}\right)\right] \\
& \left.-2\left[16^{n} f\left(\frac{y+z}{2^{n}}\right)+16^{n} f\left(\frac{y-z}{2^{n}}\right)\right]-32 \times 16^{n} f\left(\frac{x}{2^{n}}\right), t\right) \\
\geq & N_{\beta}^{\prime}\left(\varphi(x, y, z), \frac{t}{(16 \alpha)^{n \beta}}\right)
\end{aligned}
$$

for all $x, y, z \in X, t>0$, with $x \perp y, y \perp z$, and $z \perp x$ and all $n \in \mathbb{N}$. Since

$$
\lim _{n \rightarrow \infty} N_{\beta}^{\prime}\left(\varphi(x, y, z), \frac{t}{(16 \alpha)^{n \beta}}\right)=1
$$

for all $x, y, z \in X, t>0$, with $x \perp y, y \perp z$, and $z \perp x$ and all $n \in \mathbb{N}$, we get

$$
\begin{aligned}
& N_{\beta}(Q(2 x+y+z)+Q(2 x+y-z)+Q(2 x-y+z)+Q(-2 x \\
& +y+z)+Q(2 y)+Q(2 z)-8[Q(x+y)+Q(x-y)+Q(x+z) \\
& \quad+Q(x-z)]-2[Q(y+z)+Q(y-z)]-32 Q(x), t)=1
\end{aligned}
$$

for all $x, y, z \in X, t>0$ with $x \perp y, y \perp z$, and $z \perp x$. So, we get $Q: X \rightarrow Y$ is a quartic mapping.

Since $f(x)$ is an even mapping, $Q(x)$ is an even mapping, the mapping $Q: X \rightarrow Y$ is an orthogonally quartic mapping.

Letting $n \rightarrow \infty$ in (3.10), we get the inequality (3.3).

To prove the uniqueness of $Q$, let $L: X \rightarrow Y$ be another orthogonally quartic mapping satisfying (3.3). We have

$$
\begin{aligned}
& N_{\beta}(Q(x)-L(x), t) \\
& \quad=N_{\beta}\left(16^{n} Q\left(\frac{x}{2^{n}}\right)-16^{n} L\left(\frac{x}{2^{n}}\right), t\right) \\
& \quad \geq \min \left\{N_{\beta}\left(16^{n} Q\left(\frac{x}{2^{n}}\right)-16^{n} f\left(\frac{x}{2^{n}}\right), t\right), N_{\beta}\left(16^{n} f\left(\frac{x}{2^{n}}\right)-16^{n} L\left(\frac{x}{2^{n}}\right), t\right)\right\}
\end{aligned}
$$




$$
\begin{aligned}
& \geq \min \left\{N_{\beta}^{\prime}\left(\varphi\left(0, \frac{x}{2^{n}}, 0\right), \frac{\left(\alpha^{-\beta}-16^{\beta}\right) t}{2 \cdot 16^{n \beta}}\right), N_{\beta}^{\prime}\left(\varphi\left(0, \frac{x}{2^{n}}, 0\right), \frac{\left(\alpha^{-\beta}-16^{\beta}\right) t}{2 \cdot 16^{n \beta}}\right)\right\} \\
& \geq N_{\beta}^{\prime}\left(\varphi(0, x, 0), \frac{\left(\alpha^{-\beta}-16^{\beta}\right) t}{2} \frac{1}{(16 \alpha)^{n \beta}}\right),
\end{aligned}
$$

which tends to 1 as $n \rightarrow \infty$, for all $x \in X, t>0$, and all $n \in \mathbb{N}$. So $Q: X \rightarrow Y$ is unique. This completes the proof.

Corollary 3.2 Let $\theta \geq 0, p$ be a real positive number with $p>4,(X, \perp)$ be a real orthogonality vector space with norm $\|\cdot\|$ with $0<\beta \leq 1$ and $\left(\mathbb{R}, N^{\prime}\right)$ be a complete fuzzy $\beta$-normed space. If $: X \rightarrow Y$ is an even mapping satisfying $f(0)=0$ and the inequality

$$
N_{\beta}(D f(x, y, z), t) \geq N_{\beta}^{\prime}\left(\theta\left(\|x\|^{p}+\|y\|^{p}+\|z\|^{p}\right), t\right)
$$

for all $x, y, z \in X, t>0$, with $x \perp y, y \perp z$, and $z \perp x$, there is a unique orthogonally quartic mapping $Q: X \rightarrow Y$ such that

$$
N_{\beta}(f(x)-Q(x), t) \geq N_{\beta}^{\prime}\left(\varphi(0, x, 0),\left(2^{p \beta}-16^{\beta}\right) t\right)
$$

for all $x \in X, t>0$.

Proof From Theorem 3.1 by taking $\varphi(x, y, z)=\theta\left(\|x\|^{p}+\|y\|^{p}+\|z\|^{p}\right)$ for all $x, y, z \in X, t>0$, with $x \perp y, y \perp z$, and $z \perp x$, and choosing $\alpha=2^{-p \beta}$ we get the desired result.

Theorem 3.3 Assume that a mapping $: X \rightarrow Y$ is an even mapping satisfying the inequality (3.1) and $f(0)=0$ for all $x, y, z \in X, t>0$, with $x \perp y, y \perp z$, and $z \perp x$. Let $\varphi: X^{3} \rightarrow Z$ be a mapping for which there is a constant $\alpha \in \mathbb{R}$ satisfying $0<\alpha<16$ such that

$$
N_{\beta}^{\prime}\left(\varphi(x, y, z), \alpha^{\beta} t\right) \geq N_{\beta}^{\prime}\left(\varphi\left(\frac{x}{2}, \frac{y}{2}, \frac{z}{2}\right), t\right)
$$

for all $x, y, z \in X, t>0$, with $x \perp y, y \perp z$ and $z \perp x$. Then there is a unique orthogonally quartic mapping $Q: X \rightarrow Y$ such that

$$
N_{\beta}(f(x)-Q(x), t) \geq N_{\beta}^{\prime}\left(\varphi(0, x, 0),\left(16^{\beta}-\alpha^{\beta}\right) t\right)
$$

for all $x \in X, t>0$.

Corollary 3.4 Let $\theta \geq 0, p$ be a real positive number with $4>p>0,(X, \perp)$ be a real orthogonality vector space with norm $\|\cdot\|$ with $0<\beta \leq 1$ and $\left(\mathbb{R}, N^{\prime}\right)$ be a complete fuzzy $\beta$ normed space. Iff $: X \rightarrow Y$ is an even mapping satisfying the inequality (3.12) and $f(0)=0$ for all $x, y, z \in X, t>0$, with $x \perp y, y \perp z$, and $z \perp x$, there is a unique orthogonally quartic mapping $Q: X \rightarrow Y$ such that

$$
N_{\beta}(f(x)-Q(x), t) \geq N_{\beta}^{\prime}\left(\varphi(0, x, 0),\left(16^{\beta}\right) t-2^{p \beta}\right)
$$

for all $x \in X, t>0$. 


\section{Competing interests}

The authors declare that they have no competing interests.

\section{Authors' contributions}

All authors conceived of the study, participated its design and coordination, drafted the manuscript, participated in the sequence alignment, and read and approved the final manuscript.

\section{Acknowledgements}

Xiuzhong Yang was supported by the National Natural Science Foundation of China (grant No. 11371119) and all authors were supported by the Natural Science Foundation of Education Department of Hebei Province (grant No. Z2014031).

\section{Received: 20 March 2015 Accepted: 11 October 2015 Published online: 26 October 2015}

\section{References}

1. Ulam, SM: Problems in Modern Mathematics. Wiley, New York (1964)

2. Hyers, DH: On the stability of the linear functional equation. Proc. Natl. Acad. Sci. USA 27(4), 222-224 (1941)

3. Rassias, TM: On the stability of the linear mapping in Banach spaces. Proc. Am. Math. Soc. 72, 297-300 (1978)

4. Gavruta, P: A generalization of the Hyers-Ulam-Rassias stability of approximately additive mappings. J. Math. Anal. Appl. 184, 431-436 (1994)

5. Rassias, JM: Solution of the Ulam stability problem for quartic mappings. Glas. Math. 34(54), 243-252 (1999)

6. Sahoo, PK, Chung, JK: On the general solution of a quartic functional equation. Bull. Korean Math. Soc. 40(4), 565-576 (2003)

7. Lee, SH, Kim, SM, Hwang, IS: Quartic functional equations. J. Math. Anal. Appl. 307, 387-394 (2005)

8. Pinsker, AG: Sur une fonctionnelle dans l'espace de Hilbert. C. R. (Dokl.) Acad. Sci. URSS N. Ser. 20, 411-414 (1938)

9. Gudder, S, Strawther, D: Orthogonally additive and orthogonally increasing functions on vector spaces. Pac. J. Math. 58, 427-436 (1995)

10. Ger, R, Sikorska, J: Stability of the orthogonal additivity. Bull. Pol. Acad. Sci., Math. 43, 143-151 (1995)

11. Park, CG: On the stability of the orthogonally quartic functional. Bull. Iran. Math. Soc. 3(1), 63-70 (2005)

12. Rätz, J: On orthogonally additive mappings. Aequ. Math. 28, 35-49 (1985)

13. Arunkumar, M, Ravi, K, Rassias, MJ: Stability of a quartic and orthogonally quartic functional equation. Bull. Math. Anal. Appl. 3, 13-24 (2011)

14. Katsaras, AK: Fuzzy topological vector spaces II. Fuzzy Sets Syst. 12, 143-154 (1984)

15. Felbin, C: Finite-dimensional fuzzy normed linear space. Fuzzy Sets Syst. 48, 239-248 (1992)

16. Krishna, SV, Sarma, KKM: Separation of fuzzy normed linear spaces. Fuzzy Sets Syst. 63, $207-217$ (1994)

17. Kenary, HA: On the stability of a cubic functional equation in random normed spaces. J. Math. Ext. 4, 105-114 (2009)

18. Mihet, D, Radu, V: On the stability of the additive Cauchy functional equation in random normed spaces. J. Math. Anal. Appl. 343, 567-572 (2008)

19. Najati, A, Cho, Y: Generalized Hyers-Ulam stability of the Pexiderized Cauchy functional equation in non-Archimedean spaces. Fixed Point Theory Appl. 2011, 309026 (2011). doi:10.1155/2011/309026

20. Najati, A, Kang, J, Cho, Y: Local stability of the Pexiderized Cauchy and Jensen's equations in fuzzy spaces. J. Inequal. Appl. 2011, 78 (2011). doi:10.1186/1029-242X-2011-78

21. Bag, T, Samanta, SK: Finite dimensional fuzzy normed linear spaces. J. Fuzzy Math. 11(3), 687-705 (2003)

22. Cheng, SC, Mordeson, JN: Fuzzy linear operators and fuzzy normed linear spaces. Bull. Calcutta Math. Soc. 86, 429-436 (1994)

23. Karmosil, I, Michalek, J: Fuzzy metric and statistical metric spaces. Kybernetica 11, 326-334 (1975)

24. Czerwik, S: On the stability of the quadratic mapping in normed spaces. Abh. Math. Semin. Univ. Hamb. 62, 59-64 (1992)

25. Cho, Y, Park, C, Saadati, R: Functional inequalities in non-Archimedean Banach spaces. Appl. Math. Lett. 60, 1994-2002 (2010)

26. Cholewa, PW: Remarks on the stability of functional equations. Aequ. Math. 27, 76-86 (1984)

27. Diaz, JB, Margolis, B: A fixed point theorem of the alternative for contractions on generalized complete metric space. Bull. Am. Math. Soc. 74, 305-309 (1968)

28. Gordji, ME, Park, C, Savadkouhi, MB: The stability of a quartic type functional equation with the fixed point alternative Fixed Point Theory 11, 265-272 (2010)

29. Kenary, HA, Cho, Y: Stability of mixed additive-quadratic Jensen type functional equation in various spaces. Comput. Math. Appl. 61, 2704-2724 (2011)

30. Rassias, TM, Semrl, P: On the behavior of mappings which do not satisfy Hyers- Ulam stability. Proc. Am. Math. Soc. 114, 989-993 (1992)

31. Skof, F: Proprietà locali e approssimazione di operatori. Rend. Semin. Mat. Fis. Milano 53, 113-129 (1983)

32. Yang, X, Chang, L, Liu, G, Shen, G: Stability of functional equations in ( $n, \beta)$-normed spaces. J. Inequal. Appl. 2015, 112 (2015). doi:10.1186/s13660-015-0628-1

33. Cho, YJ, Rassias, TM, Saadati, R: Stability of Functional Equations in Random Normed Spaces. Springer, New York (2013)

34. Cho, YJ, Park, C, Rassias, TM, Saadati, R: Stability of Functional Equations in Banach Algebras. Springer, New York (2014)

35. Jung, SM: Hyers-Ulam-Rassias Stability of Functional Equations in Nonlinear Analysis. Springer, New York (2011)

36. Jung, SM, Popa, D, Rassias, MT: On the stability of the linear functional equation in a single variable on complete metric groups. J. Glob. Optim. 59(1), 165-171 (2014)

37. Kannappan, P: Functional Equations and Inequalities with Applications. Springer, New York (2009)

38. Lee, YH, Jung, SM, Rassias, MT: On an n-dimensional mixed type additive and quadratic functional equation. Appl. Math. Comput. 228, 13-16 (2014) 